\title{
Large-eddy simulation of turbulent collision of heavy particles in isotropic turbulence
}

\author{
Guodong Jin, ${ }^{1}$ Guo-Wei He, ${ }^{1, a)}$ and Lian-Ping Wang ${ }^{1,2}$ \\ ${ }^{1}$ State Key Laboratory for Nonlinear Mechanics, Institute of Mechanics, Chinese Academy of Sciences, \\ Beijing 100190, People's Republic of China \\ ${ }^{2}$ Department of Mechanical Engineering, University of Delaware, Newark, \\ Delaware 19716-3140, USA
}

(Received 1 October 2009; accepted 30 March 2010; published online 20 May 2010)

\begin{abstract}
The small-scale motions relevant to the collision of heavy particles represent a general challenge to the conventional large-eddy simulation (LES) of turbulent particle-laden flows. As a first step toward addressing this challenge, we examine the capability of the LES method with an eddy viscosity subgrid scale (SGS) model to predict the collision-related statistics such as the particle radial distribution function at contact, the radial relative velocity at contact, and the collision rate for a wide range of particle Stokes numbers. Data from direct numerical simulation (DNS) are used as a benchmark to evaluate the LES using both a priori and a posteriori tests. It is shown that, without the SGS motions, LES cannot accurately predict the particle-pair statistics for heavy particles with small and intermediate Stokes numbers, and a large relative error in collision rate (up to 60\%) may arise when the particle Stokes number is near $\mathrm{St}_{K}=0.5$. The errors from the filtering operation and the SGS model are evaluated separately using the filtered-DNS (FDNS) and LES flow fields. The errors increase with the filter width and have nonmonotonic variations with the particle Stokes numbers. It is concluded that the error due to filtering dominates the overall error in LES for most particle Stokes numbers. It is found that the overall collision rate can be reasonably predicted by both FDNS and LES for $\mathrm{St}_{K}>3$. Our analysis suggests that, for $\mathrm{St}_{K}<3$, a particle $\mathrm{SGS}$ model must include the effects of SGS motions on the turbulent collision of heavy particles. The spectral analysis of the concentration fields of the particles with different Stokes numbers further demonstrates the important effects of the small-scale motions on the preferential concentration of the particles with small Stokes numbers. (C) 2010 American Institute of Physics.
\end{abstract}

[doi:10.1063/1.3425627]

\section{INTRODUCTION}

Particle-laden turbulent flows are encountered in a wide range of engineering and environmental problems; ${ }^{1,2}$ for example, pollutant or aerosol particle dispersion, warm rain droplet formation in the atmosphere, and fluidization and turbulent mixing in combustion processes. ${ }^{3,4}$ Understanding the hydrodynamics of such flows is the basis for many applications in engineering design, environmental protection, and weather forecasting. Particularly, turbulence-mediated collision of heavy particles is vital to efficiency and conversion rates in these processes. ${ }^{5}$

Large-eddy simulation (LES) has emerged as a promising tool for simulating turbulent flows in general and, in recent years, has also been applied to particle-laden turbulence with some successes. ${ }^{6}$ The motion of heavy particles is much more complicated than fluid elements, and therefore, LES of turbulent flow laden with heavy particles encounters new challenges. In LES, only large-scale eddies are explicitly resolved and the effects of unresolved, small-scale or subgrid scale (SGS) eddies on the large-scale eddies are parametrized. The SGS turbulent velocity field is not available.

\footnotetext{
${ }^{a)}$ Author to whom correspondence should be addressed. Electronic mail: hgw@1nm.imech.ac.cn and guoweihe@yahoo.com. Telephone: 86-1082543969. Fax: 86-10-82543408.
}

The effects of SGS turbulent velocity fields on particle motions have been studied in channel flows or isotropic turbulent flows by Yeh and Lei, ${ }^{7}$ Wang and Squires, ${ }^{8}$ Armenio, Piomelli, and Fiorotto, ${ }^{9}$ Yamamoto et al. ${ }^{10}$ Kuerten, ${ }^{11}$ Shotorban and Mashayek, ${ }^{12,13}$ Fede and Simonin, ${ }^{14}$ Berrouk et al. ${ }^{15}$ Bini and Jones, ${ }^{16}$ Pozorski and Apte, ${ }^{17}$ among others. The Eulerian deconvolution method ${ }^{11,12}$ or Lagrangian stochastic approach $^{13,15,17}$ is usually used to model the effects of SGS motions on particle dispersion. The missing of SGS turbulent velocity does not pose a serious problem for quantifying the properties that are mainly governed by large-scale turbulent eddies, i.e., single-particle statistics such as oneparticle dispersion coefficient. ${ }^{7-9}$ However, the turbulent collision of heavy particles is a small-scale process, where the small-scale velocity field plays an important role. The collision-related relative motions and pair distribution of heavy particles with the Stokes numbers $\left(\mathrm{St}_{K} \equiv \tau_{p} / \tau_{K}\right.$, i.e., the ratio of heavy particle response time to flow Kolmogorov time) on the order of 1 is mainly determined by the smallscale eddies. ${ }^{18-20}$ These particle-pair statistics and thus collision rate of heavy particles are unlikely to be properly simulated in LES. This is one of the main challenges in LES of particle-laden turbulent flows. Another issue is that the flow field in LES is more coherent in space and more correlated in time than either the fully resolved flow field or direct nu- 
merical simulation (DNS) one due to the filtering operation and overdissipation of the commonly used eddy viscosity SGS model. ${ }^{21-23}$ The flow field from the LES with an eddy viscosity SGS model may not recover the properties especially needed for collision-rated statistics of heavy particles.

Fede and Simonin ${ }^{14}$ studied the effects of SGS turbulent motions on particle-pair statistics of nonsettling, monodispersed heavy particles in an isotropic turbulent flow field. DNS was used to generate a turbulent flow at Taylor microscale Reynolds number $\operatorname{Re}_{\lambda}=34$.1. The filtered-DNS (simply denoted as FDNS hereafter) flow field was obtained from the DNS one by filtering the small-scale fluid motions at different cutoff scales. One-way coupling was assumed and particles were treated as perfect-elastic hard spheres. They focused on the effects of SGS motions on both one- and twoparticle statistics of heavy particles. They concluded that the effects of SGS motions must be included when the SGS Stokes number $\tau_{p} / \delta \tau_{f @ p}$ is less than 5.0, where $\delta \tau_{f @ p}$ is the SGS Lagrangian integral time scale seen by heavy particles. A very interesting result is that the effect of filtering leads to a reduction in preferential concentration if $\tau_{p}<0.5 \delta \tau_{f @ p}$, but an increase in the preferential concentration if $\tau_{p}$ $>0.5 \delta \tau_{f @ p}$. For the case of $\tau_{p}<0.5 \delta \tau_{f @ p}$, the eddies at the dissipation scales play an important role in particle clustering. Filtering reduces the intensity of the dissipation-scale eddies and thus reduces the level of preferential concentration. For the case of $5 \delta \tau_{f @ p}>\tau_{p}>0.5 \delta \tau_{f @ p}$, the particles respond to the eddies ranging from the dissipation-scale eddies to the larger-scale ones. By removing the dissipationscale eddies, the larger-scale eddies may actually become more coherent in space and better correlated in time, although the maximum vorticity intensity is reduced. This implies that the actual level of preferential concentration is governed not only by the vorticity intensity but also by the vorticity distribution. Fede et l. $^{24}$ then developed a onepoint stochastic Lagrangian model to recover the SGS motions seen by heavy particles. The model predicts a good match to DNS data for the particle kinetic energy.

The collision-related statistics has not been quantitatively compared and assessed simultaneously using DNS, FDNS, and LES in previous studies. The aim of this paper is to investigate the effects of the spectral eddy viscosity SGS model and the SGS fluid motions on particle-pair statistics such as radial distribution function at contact, radial relative velocity at contact, and collision rate. By understanding these effects, we hope to develop some insights into the dynamic interaction of heavy particles with SGS flow fields, as well as a guidance on a better particle SGS model aimed at improving particle-pair statistics in LES. This paper is organized as follows. An overview of the governing equations and simulation methods is given in Sec. II The numerical results about the effects of SGS motions on collision-related statistics of heavy particles are presented in Sec. III. Conclusions on LES of turbulent collision of heavy particles can be found in Sec. IV.

\section{GOVERNING EQUATIONS AND SIMULATION METHODS}

The equations governing fluid flow and motion of heavy particles are described in this section, along with our numerical methods.

\section{A. DNS method}

The Navier-Stokes equations for isotropic turbulence are

$$
\begin{aligned}
& \frac{\partial \boldsymbol{u}}{\partial t}=\boldsymbol{u} \times \boldsymbol{\omega}-\nabla\left(\frac{p}{\rho}+\frac{1}{2} u^{2}\right)+\nu \nabla^{2} \boldsymbol{u}+\boldsymbol{f}(\boldsymbol{x}, t), \\
& \nabla \cdot \boldsymbol{u}=0,
\end{aligned}
$$

where $\boldsymbol{u}$ denotes the velocity, $\boldsymbol{\omega}=\nabla \times \boldsymbol{u}$ is the vorticity, $p$ is the pressure, $\rho$ is the fluid density, and $\nu$ is the fluid kinematical viscosity. The flow is driven and maintained by a random forcing $f(x, t)$, which is nonzero only at low wavenumbers in Fourier space $|\boldsymbol{k}|<\sqrt{8} .^{25,26}$

The DNS of isotropic turbulence is performed using a standard pseudospectral method on $N^{3}$ grids covering a periodic box of side $L=2 \pi$. Here, $N=256$. In Fourier space, Eqs. (1) and (2) can be represented as $\left(k \leq k_{\max }\right)$,

$$
\left(\frac{\partial}{\partial t}+\nu k^{2}\right) \hat{\boldsymbol{u}}(\boldsymbol{k}, t)=\boldsymbol{P}(\boldsymbol{k}) F(\boldsymbol{u} \times \boldsymbol{\omega})+\hat{\boldsymbol{f}}(\boldsymbol{k}, t),
$$

where $\hat{\boldsymbol{u}}(\boldsymbol{k}, t)$ is the Fourier coefficient or the fluid velocity in Fourier space, $F$ denotes a Fourier transformation, the projection tensor $\boldsymbol{P}=\left\{P_{j m}\right\}=\delta_{j m}-k_{j} k_{m} / k^{2} \quad(j, m=1,2,3)$ projects $F(\boldsymbol{u} \times \boldsymbol{\omega})$ onto the plane normal to $\boldsymbol{k}$ and eliminates the pressure term in Eq. (1) with the aid of the continuity equation (2), and $k_{\max }$ is the maximum wavenumber.

The wavenumber components in Fourier space are $k_{j}$ $=n_{j}(2 \pi / L)$, where $n_{j}=-N / 2, \ldots,-1,0,1, \ldots, N / 2-1$ for $j$ $=1,2,3$. Aliasing errors are removed using the two-thirds truncation method. The spatial resolution is monitored by the value of $k_{\max } \eta$, where $\eta$ is the Kolmogorov length scale. The value of $k_{\max } \eta$ is typically larger than 1.1 in the present simulation. The Fourier coefficients are advanced in time using a second-order Adams-Bashforth method for the nonlinear term and an exact integration for the linear viscous term. The time step is chosen to ensure that the CourantFriedrichs-Lewy number is less than 0.5 for numerical stability and accuracy. ${ }^{26,27}$

\section{B. FDNS velocity fields}

The FDNS velocity field is obtained from the DNS velocity field by truncating the Fourier coefficients larger than the cutoff wavenumber $k_{\mathrm{cf}}$,

$$
\widetilde{\mathbf{u}}(\boldsymbol{x}, t)=\sum_{|\boldsymbol{k}|=k_{0}}^{k_{\mathrm{cf}}} \hat{\boldsymbol{u}}(\boldsymbol{k}, t) e^{i \boldsymbol{k} \cdot \boldsymbol{x}},
$$

where $\widetilde{\boldsymbol{u}}(\boldsymbol{x}, t)$ is the filtered velocity in physical space and $k_{\mathrm{cf}}$ is the cutoff wavenumber in FDNS. $k_{0}=1$ is the lowest wavenumber. FDNS can be regarded as an ideal LES to study the 
effects of SGS eddies on the statistics of particle motions since FDNS does not contain any eddy viscosity SGS model errors. $^{14}$

\section{LES method}

The LES of isotropic turbulence is performed on the coarser grids using the same pseudospectral method and large-scale forcing scheme as DNS. The governing equation in LES is given by $\left(|\boldsymbol{k}| \leq k_{c}\right.$, where $k_{c}$ is the maximum wavenumber in LES)

$$
\left\{\frac{\partial}{\partial t}+\left[\nu+\nu_{e}\left(k \mid k_{c}\right)\right] k^{2}\right\} \hat{\overline{\boldsymbol{u}}}(\boldsymbol{k}, t)=\boldsymbol{P}(\boldsymbol{k}) F(\overline{\boldsymbol{u}} \times \overline{\boldsymbol{\omega}})+\hat{\boldsymbol{f}}(\mathbf{k}, t),
$$

where $\overline{\boldsymbol{u}}$ and $\overline{\boldsymbol{\omega}}$ are the resolved velocity and vorticity in physical space, respectively. The term $\nu_{e}\left(k \mid k_{c}\right) k^{2} \hat{\bar{u}}(\boldsymbol{k}, t)$ on the left-hand side models the net effects of SGS motions on the resolved motions. The spectral eddy viscosity SGS model $^{21,22}$ is used in this paper, given by

$$
\nu_{e}\left(k \mid k_{c}\right)=\nu_{e}^{+}\left(k / k_{c}\right) \sqrt{\frac{E\left(k_{c}\right)}{k_{c}}},
$$

with

$$
\nu_{e}^{+}\left(k / k_{c}\right)=C_{K}^{-3 / 2}\left[0.441+15.2 \exp \left(-3.03 k_{c} / k\right)\right] .
$$

The spectral viscosity $\nu_{e}\left(k \mid k_{c}\right)$ depends on the wavenumber $k$, the maximum wavenumber $k_{c}$ as well as $E\left(k_{c}\right)$, the value of the energy spectrum function at $k_{c}$. Here, $E\left(k_{c}\right)$ in Eq. (6) is dynamically evaluated from the LES fluid field. $C_{K}=2.5$ is taken in this paper.

\section{Motion of inertial particles}

The dispersed phase is composed of $N_{p}$ spherical heavy particles with a diameter smaller than the Kolmogorov length scale $\eta, d_{p}=0.5 \eta$. In general, there are several different forces acting on a heavy particle suspended in a nonuniform and unsteady turbulent flow field. ${ }^{28}$ When the ratio of particle density to fluid density is much larger than 1 $\left(\rho_{p} / \rho_{f} \gg 1\right)$, the equation of motion for a heavy particle can be approximated as ${ }^{26}$

$$
\begin{aligned}
& \frac{d \boldsymbol{x}_{p}(t)}{d t}=\boldsymbol{v}_{p}(t), \\
& \frac{d \boldsymbol{v}_{p}(t)}{d t}=\frac{\boldsymbol{u}\left[\boldsymbol{x}_{p}(t), t\right]-\boldsymbol{v}_{p}(t)+\boldsymbol{w}_{0}}{\tau_{p}},
\end{aligned}
$$

where $\boldsymbol{x}_{p}(t)$ and $\boldsymbol{v}_{p}(t)$ are the particle position and velocity at time $t, \boldsymbol{w}_{0}$ is the particle terminal or settling velocity under gravity in otherwise quiescent fluid, $\boldsymbol{w}_{0}=\boldsymbol{g} \tau_{p}, \tau_{p}$ is the particle Stokes response time, and $\boldsymbol{g}$ is the gravitational acceleration. The particle terminal velocity is set to $\boldsymbol{w}_{0}=\left(-v_{K}, 0,0\right)$ in this study, where $v_{K}$ is the Kolmogorov velocity obtained from DNS. The linear Stokes drag force is assumed due to the low particle Reynolds number, $\boldsymbol{u}\left[\boldsymbol{x}_{p}(t), t\right]$ is the fluid velocity seen by a heavy particle which is obtained from the DNS, FDNS, and LES flow fields, respec-
TABLE I. The parameters in DNS and LES.

\begin{tabular}{lcc}
\hline \hline Method & DNS $\left(256^{3}\right)$ & LES $\left(64^{3}\right)$ \\
\hline Reynolds number & 102.05 & $\ldots$ \\
rms fluid velocity $u^{\prime}$ & 19.34 & 18.52 \\
(Effective) dissipation rate & 3771.4 & 3434.8 \\
Minimum length scale & 0.0135 & 0.0238 \\
Minimum time scale & 0.0037 & 0.0055 \\
Minimum velocity scale & 3.6272 & 4.327 \\
Eulerian integral time scale & 0.050 & 0.056 \\
Molecular viscosity & 0.0488 & 0.0488 \\
\hline \hline
\end{tabular}

tively, by a six-point Lagrangian interpolation scheme in each direction. ${ }^{27}$ The equation of motion [Eq. (9)] is integrated with a fourth-order Adams-Bashforth method for the particle velocity and then a fourth-order Adams-Moulton method for the particle location in Eq. (8). The particles are assumed to have identical size and density, and each moves independent of others (i.e., the so-called ghost particle model). This is appropriate for turbulent geometric collision. ${ }^{29,30}$ All particle pairs and collision search are conducted using the efficient cell-index method and the concept of linked lists. ${ }^{18,31}$

\section{NUMERICAL RESULTS}

\section{A. Statistics of DNS and LES flow fields}

Table I lists Eulerian statistics of the DNS and LES flow fields in this study. The root mean square (rms) of turbulent fluctuation velocity $u^{\prime}$ and the average dissipation rate $\varepsilon$ are computed from the three dimensional turbulent energy spectrum function shown in Fig. 1,

$$
u^{\prime}=\sqrt{\frac{1}{3}\left\langle u_{i} u_{i}\right\rangle}=\sqrt{\frac{2}{3} \int_{k_{0}}^{k_{\max }} E(k) d k},
$$

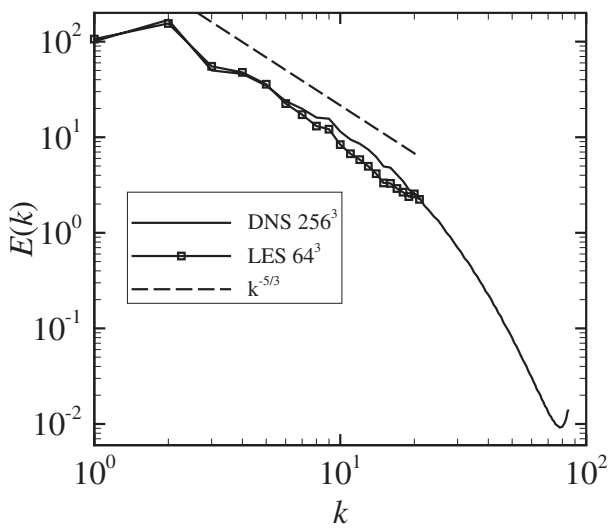

FIG. 1. The energy spectra of the simulated flows in $256^{3}$ DNS and $64^{3}$ LES. 


$$
\varepsilon=\int_{k_{0}}^{k_{\max }} 2 \nu k^{2} E(k) d k .
$$

In LES, the molecular viscosity coefficient $\nu$ in Eq. (11) is replaced by the effective viscosity $\nu+v_{e}\left(k \mid k_{c}\right)$ to calculate the effective dissipation rate,

$$
\varepsilon_{e}=\int_{k_{0}}^{k_{c}} 2\left[\nu+v_{e}\left(k \mid k_{c}\right)\right] k^{2} E(k) d k,
$$

which represents the sum of the molecular dissipation rate of large-scale modes and the dissipation rate of the SGS eddies. The dissipation rate in LES $\left(64^{3}\right)$ listed in Table I is the effective one. The actual or molecular dissipation rate of the large-scale motion is 1626.6 .

As the spectral eddy viscosity coefficient $v_{e}\left(k \mid k_{c}\right)$ varies with the wavenumber, an average SGS viscosity coefficient can be estimated using the enstrophy spectrum $2 k^{2} E(k)$ as weight factors,

$$
\bar{\nu}_{\mathrm{SGS}}=\frac{\int_{k_{0}}^{k_{c}} 2 v_{e}\left(k / k_{c}\right) k^{2} E(k) d k}{\int_{k_{0}}^{k_{c}} 2 k^{2} E(k) d k} .
$$

This average eddy viscosity coefficient in LES, $\bar{\nu}_{\mathrm{SGS}}$ $=0.0543$, is only slightly larger than the molecular viscosity coefficient used in DNS, $\nu=0.0488$. This could be due to the moderate flow Reynolds number realized in DNS, given that the same large-scale flow forcing and flow domain size are applied in LES and DNS. The smallest (Kolmogorov) length, time, and velocity scales in DNS are

$$
\eta=\left(\nu^{3} / \varepsilon\right)^{0.25}, \quad \tau_{K}=(\nu / \varepsilon)^{0.5}, \quad v_{K}=(\varepsilon \nu)^{0.25} .
$$

In order to obtain the minimum characteristic scales in LES flow field, the effective viscosity $\left(\nu+\bar{\nu}_{\mathrm{SGS}}\right)$ and effective dissipation rate $\varepsilon_{e}$ are used to replace the molecular viscosity $\nu$ and molecular dissipation rate $\varepsilon$, respectively, in Eq. (14) to represent the smallest characteristic length, time, and velocity scales as

$$
\begin{aligned}
& \eta_{e}=\left[\frac{\left(\nu+\bar{\nu}_{\mathrm{SGS}}\right)^{3}}{\varepsilon_{e}}\right]^{0.25}, \quad \tau_{K, e}=\left[\frac{\left(\nu+\bar{\nu}_{\mathrm{SGS}}\right)}{\varepsilon_{e}}\right]^{0.5}, \\
& \mathrm{v}_{K, e}=\left[\varepsilon_{e}\left(\nu+\bar{\nu}_{\mathrm{SGS}}\right)\right]^{0.25} .
\end{aligned}
$$

Ideally, $\varepsilon_{e}=\varepsilon$ if there was no SGS model error in LES. Figure 1 plots the resulting energy spectra of DNS and LES flow fields in log-log coordinates. It is shown that the energy spectrum of LES flow field decays faster than that of DNS flow field at high wavenumbers. This is due to the overdissipation in the spectral eddy viscosity SGS model. In this study, the cutoff wavenumber in FDNS is taken as $k_{\mathrm{cf}}=21$, corresponding to the LES at the grid resolution of $64^{3}$.

\section{B. Preferential concentration of heavy particles}

It is well known that, unlike passive tracers, heavy particles are distributed nonuniformly in a turbulent flow field due to their interaction with vortical and straining motions of local flows. Maxey ${ }^{32}$ developed an asymptotic analysis of the preferential concentration of particles with weak inertia. If we consider the particle distribution from an Eulerian viewpoint and the particle velocity field is then defined as $\mathbf{v}(\mathbf{x}, t)$, its divergence field is

$$
\nabla \cdot \mathbf{v}=-\tau_{p} \frac{\partial u_{j}}{\partial x_{i}} \frac{\partial u_{i}}{\partial x_{j}}=-\tau_{p}\left(s_{i, j}^{2}-\frac{1}{2} \omega^{2}\right),
$$

where $\omega=|\nabla \times \mathbf{u}|$ and $s_{i j}=\frac{1}{2}\left(\partial u_{i} / \partial x_{j}+\partial u_{j} / \partial x_{i}\right)$ are the local fluid vorticity and strain rate tensor, respectively. Equation (16) indicates that particle velocity field is compressible even in an incompressible fluid velocity field and particles accumulate in regions of low vorticity and high strain rate.

Figure 2 shows the preferential concentration of heavy particles at different particle Stokes numbers in a DNS flow field. $N_{p}=1.2 \times 10^{6}$ heavy particles are randomly released into a statistically stationary flow field. The spatial distribution of particles evolves with time and particle preferential concentration develops in the flow field. After about four times of the Eulerian integral time scale, the accumulation is balanced by randomly turbulent mixing and the preferential concentration reaches its quasiequilibrium state. The particle terminal velocity is $\boldsymbol{w}_{0}=\left(-v_{K}, 0,0\right)$ for all kinds of particles, where $v_{K}$ is the Kolmogorov velocity. The background shows the contour of local flow vorticity at $z=\pi$ obtained from DNS $\left(256^{3}, \operatorname{Re}_{\lambda}=102.05\right)$. The locations of all particles in a slice with a thickness of $2 \pi / 256$ centered at $z$ $=\pi$ are projected onto the $x-y$ plane. Three Stokes numbers are considered: $\mathrm{St}_{K}=0.1,1.0,10.0$. The degree of nonuniformity depends on the particle Stokes numbers. For the Stokes number $\mathrm{St}_{K}=0.1$, the heavy particle response time is much shorter than the Kolmogorov time scale; therefore, particles respond quickly to the changes of the flow field and nearly follow the fluid motion. In this case, large-scale fluid motion serves to efficiently disperse the particles. However, even at $\mathrm{St}_{K}=0.1$, the inertial bias is noticeable and more particles are found in the regions of low flow vorticity [see the dark regions in Fig. 2(a)]. For $\mathrm{St}_{K}=1$, particle motion is strongly affected by small-scale eddies and particles are centrifuged out of the vortical structures, leading to a strong nonuniformity [see Fig. 2(b)]. As heavy particle response time increases, particles respond to the eddies with larger time scales relative to the Kolmogorov eddies. The level of accumulation starts to drop when $\mathrm{St}_{K}>1$. At $\mathrm{St}_{K}=10$, particles accumulate into large-scale structures and are mixed by small eddies. Therefore, particles return back to a more uniform distribution [see Fig. 2(c)]. Since the particles have a fixed terminal velocity of $v_{K}$ in the negative $x$ direction, patches of particles aligned in the vertical direction are visible in Figs. 2(b) and 2(c) as a result of the preferential sweeping. ${ }^{26}$

In general, the particle concentration field contains more large-scale structures as the Stokes number increases. This is demonstrated in Fig. 3, which plots the energy spectra of concentration fluctuations at the Stokes numbers $\mathrm{St}_{K}=0.1$, $0.5,1.0,3.0,5.0$, and 10.0, ranging from small to large inertial particles. The particle concentration $C(\boldsymbol{x}, t)$ is defined as the number of particles in a local cell of center $x$. In the present calculations, $128^{3}$ coarse grids are used to smooth the particle concentration field, although $256^{3}$ fine grids are used 


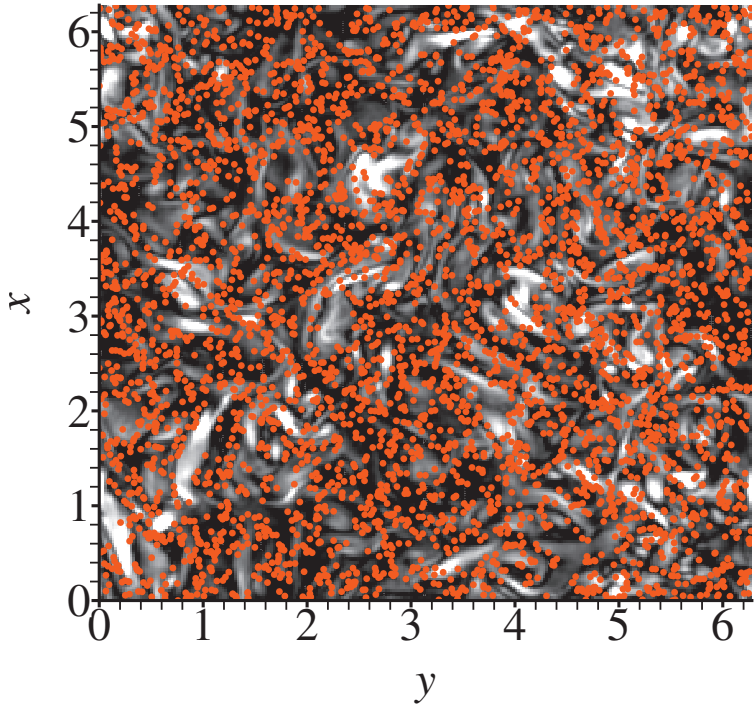

(a) $\mathrm{St}_{\mathrm{K}}=0.1$
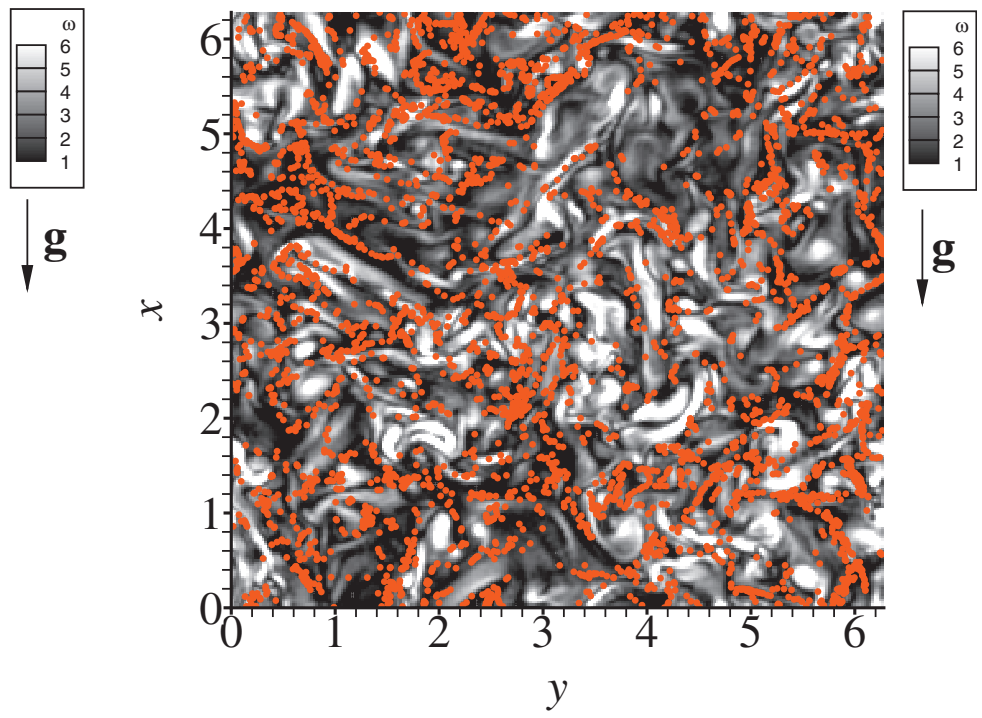

(b) $\mathrm{St}_{\mathrm{K}}=1.0$

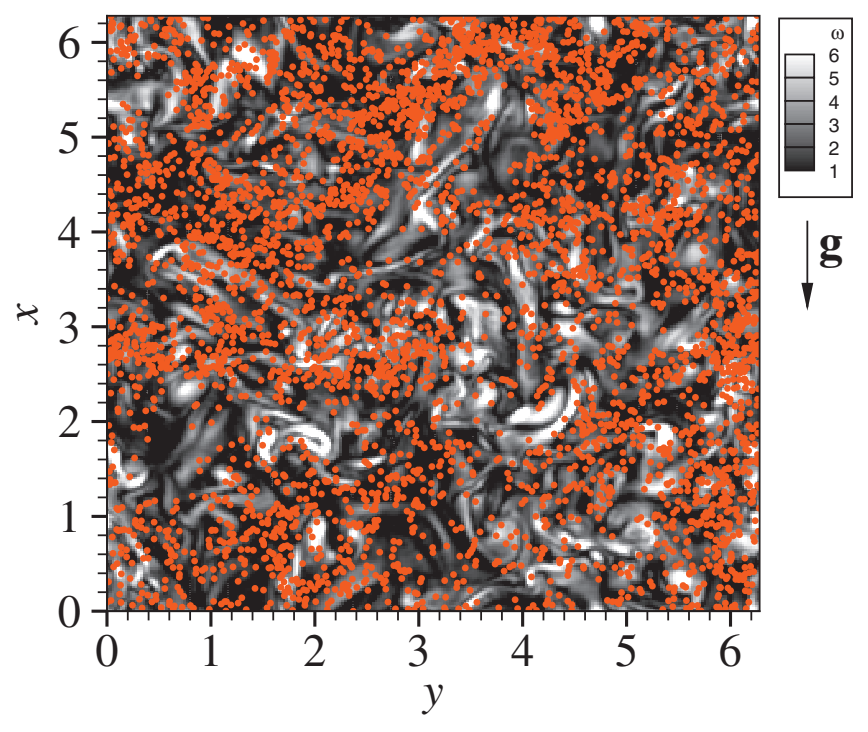

(c) $\mathrm{St}_{\mathrm{K}}=10.0$

FIG. 2. (Color) The preferential concentration of particles with different Stokes numbers in a slice centered at $z=\pi$ with a thickness of $2 \pi / 256$. The background is the vorticity contour at $z=\pi$ of DNS flow field, which is normalized by the average vorticity. The arrow below the legend represents the direction of gravity and the particle terminal velocity is set to $\boldsymbol{w}_{0}=\left(-v_{K}, 0,0\right)$, where $v_{K}$ is the Kolmogorov velocity. For a small Stokes number in (a), the particles almost follow the trajectories of fluid particles and uniformly distribute in the flow field. For a large Stokes number in (c), the particles are too heavy to respond to the eddies and also uniformly distribute in the flow field. For an intermediate Stokes number in (b), the preferential concentration is most obvious.

for the flow field in DNS. The difference between the particle concentration $C(\boldsymbol{x}, t)$ at time $t$ and the initial concentration $C(\boldsymbol{x}, 0)$ is defined as the concentration fluctuations, which largely remove the statistical noises in practical computations. Since the probability distribution function (PDF) of the initial concentration $C(\boldsymbol{x}, 0)$ is set to be a Poisson distribution, the difference $C(\boldsymbol{x}, t)-C(\boldsymbol{x}, 0)$ measures the fluctuations of concentration $C(\boldsymbol{x}, t)$ relative to the uniform concentration $C(\boldsymbol{x}, 0)$. When the particle concentration field becomes statistically stationary, the concentration fluctuations are trans- formed into the Fourier space and the squared magnitudes of the modes in each wavenumber shell within the two radii between $k-0.5$ and $k+0.5$ are summed to yield the concentration energy spectrum $E_{c}(k)$, where $k=1,2,3, \ldots$. Ten time frames with a time interval equal to $0.2 T_{E}$ are used to obtain an average energy spectrum for each Stokes number, where $T_{E}$ is the Eulerian integral time. The area under each curve is equal to $\left\langle C^{2}\right\rangle-\left\langle C^{2}(0)\right\rangle=\int_{1}^{\infty} E_{c}(k) d k$, where $\left\langle C^{2}(0)\right\rangle$ denotes the concentration variance of heavy particles at initial time. It measures the degree of nonuniformity of the particle con- 


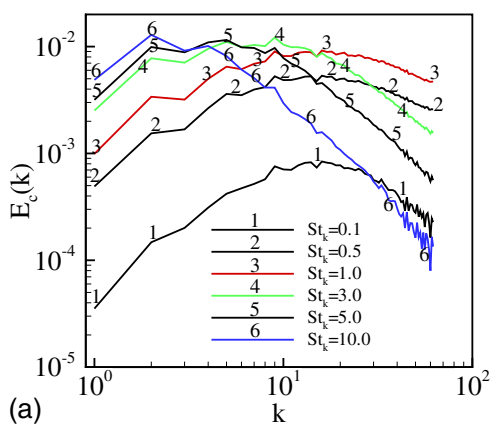

(a)

$\mathrm{k}$

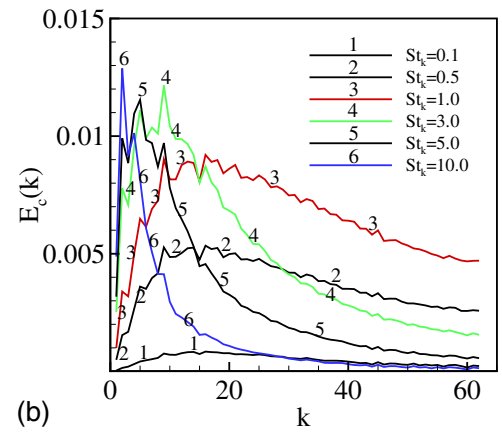

FIG. 3. (Color online) Wavenumber spectra of particle concentration fields of heavy particles with different Stokes numbers. (a) Logarithmic scales; (b) linear scales. centration for a given Stokes number. This area increases with $\mathrm{St}_{k}$, reaches a maximum at $\mathrm{St}_{k}=O(1)$, and then decreases with $\mathrm{St}_{k}$. It is observed from Fig. 3(a) that as the Stokes numbers increase, the peaks of concentration spectra move from larger wavenumbers to smaller ones with more concentration energy contained at small wavenumbers. The peak wavenumber gives an indication of the length scale at which these eddies contribute most significantly to the concentration field. Figure 3(b) shows that the small scales contain more concentration energy than the large ones for $\mathrm{St}_{K}$ $\leq 3$, while the small scales contain less concentration energy than the large ones for $S t_{K}>3$. Therefore, Fig. 3 implies that the small-scale eddies are important to the particle concentration for $\mathrm{St}_{K} \leq 3$, and simply neglecting small-scale eddies in the conventional LES may lead to incorrect predictions. A particle SGS model to account for the effects of fluid motions at small scales on particle motions is needed in LES.

In order to study the effects of average particle number concentration on the energy spectrum of particle concentration field, we doubled the number of particles to $N_{p}=2.4$ $\times 10^{6}$ while keeping the same mesh resolution, i.e., $128^{3}$ when defining the local number concentration. The energy spectra of nondimensional particle concentration fields, $\hat{E}_{c}(k)$, are plotted in Fig. 4, where the nondimensional particle concentration field is defined as $C(\boldsymbol{x}, t) /\langle C\rangle$ and $\langle C\rangle$ $=N_{p} /(2 \pi)^{3}$ is the volume-averaged particle concentration. In Fig. 4, the thin solid lines with symbols are the results obtained with $N_{p}=1.2 \times 10^{6}$ and the thick dashed lines are the

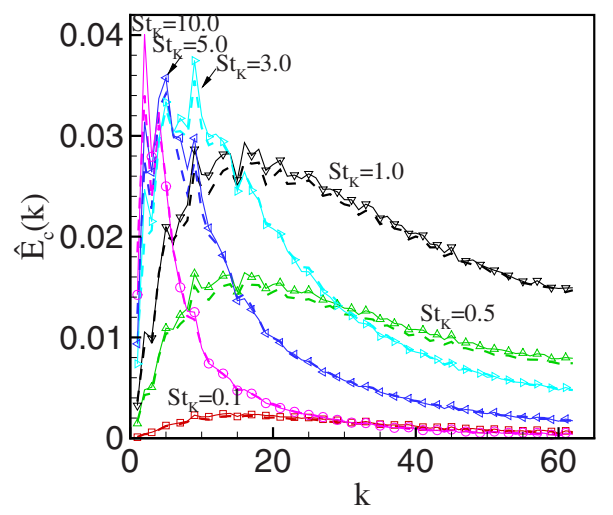

FIG. 4. (Color online) Wavenumber spectra of nondimensional particle concentration. The thin solid lines with symbols are the results obtained with $N_{p}=1.2 \times 10^{6}$ and the thick dashed lines are the results with $N_{p}=2.4 \times 10^{6}$. The wavenumber spectra for the two particle numbers almost collapse for each Stokes number considered. results obtained with $N_{p}=2.4 \times 10^{6}$. We observe that the wavenumber spectra of the nondimensional particle concentration are not sensitive to $N_{p}$ for all Stokes numbers considered, although the curves obtained with the larger $N_{p}$ are smoother as expected.

\section{Radial distribution function}

The nonuniformity of particle concentration shown in Fig. 2 has an important consequence on turbulent collision rates, which can be quantified in terms of the radial distribution function. ${ }^{18,33}$ For monodispersed particle system in a statistically isotropic flow field, the radial distribution function $g(r)$ is defined as the ratio of the number density of particle pairs with interparticle distance from $r$ to $r+d r$, $N_{\text {pair }}(r) / V_{s}$, to the average number density of particle pairs in the flow domain,

$$
g(r)=\frac{N_{\text {pair }}(r)}{V_{s}} / \frac{\frac{1}{2} N_{p}\left(N_{p}-1\right)}{(2 \pi)^{3}},
$$

where $V_{s}=\frac{4}{3} \pi\left[(r+d r)^{3}-r^{3}\right]$ is the volume of the spherical shell, $N_{\text {pair }}(r)$ is the number of particles in the shell, and $N_{p}\left(N_{p}-1\right) / 2$ is the total possible particle pairs for $N_{p}$ monodispersed particles. ${ }^{18}$

For a uniformly distributed system, the radial distribution function approaches 1. Figure 5 shows the transient variation of the radial distribution function of particles with $\mathrm{St}_{K}=1.0$ in the $256^{3}$ DNS flow field. At beginning, the particles are uniformly distributed so that $g(r / R)=1$, where $R$ is

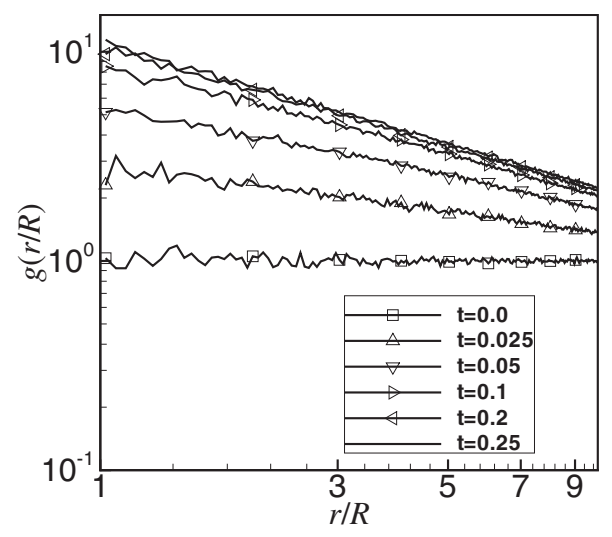

FIG. 5. Variation in the radial distribution function $g(r / R)$ with the separation distance at different times in flow field of $256^{3} \mathrm{DNS}$, where the particle Stokes number $\mathrm{St}_{K}=1.0 . g(r / R)$ exhibits a power-law scaling with $r$. 


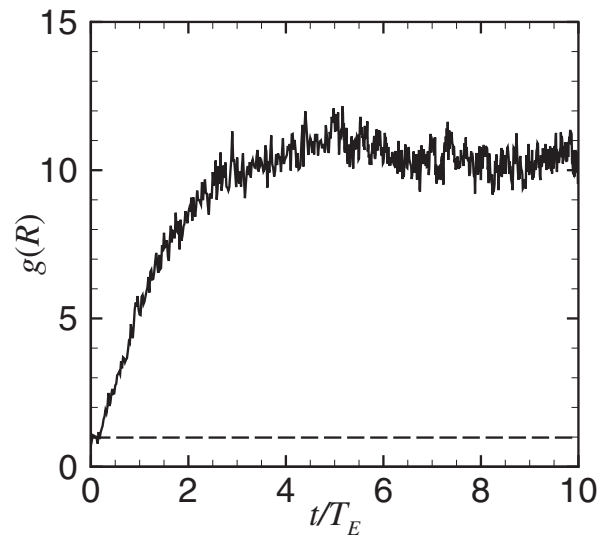

FIG. 6. Variation in the radial distribution function at contact $g(R)$ with time in flow field of $256^{3} \mathrm{DNS}$ and the particle Stokes number $\mathrm{St}_{K}=1.0$. The flow reaches a statistically steady state with fluctuations after about four times of the Eulerian integral time scale, $T_{E}=0.05$. The fluctuation is related to the finite numbers of particles, which is shown in Fig. 7.

the geometric collision radius, $R=d_{p}$ for monodispersed particles, and $d_{p}$ is the particle diameter. As time increases, particle concentration deviates from the initially uniform distribution and $g(r / R)$ increases monotonically with time. Finally, the concentration field approaches a statistically stationary state after about four times of the Eulerian integral time scale $T_{E}$. At the quasiequilibrium, we found that $g(r / R)$ follows a power-law scaling with $r / R$, consistent with previous results. ${ }^{29}$ It is also observed in Fig. 5 that $g(r / R)$ still follows the power-law scaling even in the case of sedimentation.

For geometric collision rate, the radial distribution function at contact, i.e., $g(r / R=1)$, is of interest. ${ }^{33}$ Figure 6 shows the variation in $g(r / R=1)$ with time. At long times, $g(r / R)$ fluctuates with time due to the finite number of particles and turbulent mixing. The magnitude of fluctuation decreases as the particle number increases. Figure 7 shows that the magnitude of fluctuation for $10^{6}$ particles is smaller than that of $4 \times 10^{5}$ particles in the $64^{3}$ LES flow field where the particle Stokes number $\mathrm{St}_{K}=1.3$. Some of the fluctuations

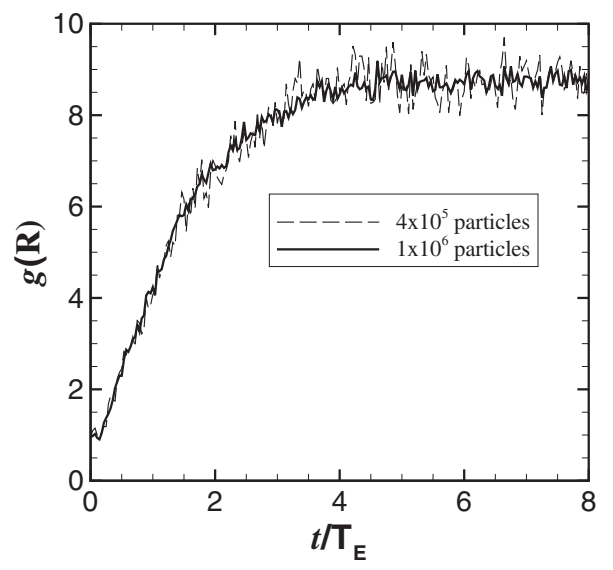

FIG. 7. The effect of particle numbers on the magnitude of fluctuation of $g(R)$ in the flow of $64^{3} \mathrm{LES}, \mathrm{St}_{K}=1.3$. The fluctuation magnitude of $g(R)$ for $10^{6}$ particles is much smaller than that for $4 \times 10^{5}$. The Eulerian integral time scale of LES flow field $T_{E}=0.056$. result from the random large-scale forcing. The time averaged value of $g(R)$ does not depend on the used particle number. The results reported in the following are based on $4 \times 10^{5}$ particles and are averaged over time after the quasiequilibrium state is obtained.

\section{Effects of filter width on the collision-related statistics}

The collision rate of monodispersed particles is formulated as ${ }^{18}$

$$
\left\langle\dot{N}_{c}\right\rangle=2 \pi R^{2}\left\langle\left|w_{r}(R)\right|\right\rangle\langle g(R)\rangle \frac{n_{0}^{2}}{2},
$$

where $n_{0}=N_{p} /(2 \pi)^{3}$ is the average particle number density, $N_{p}$ is the total particle number in the flow domain, $\left\langle\left|w_{r}(R)\right|\right\rangle$ is the averaged radial relative velocity of collision pairs at contact,

$$
\left\langle\left|w_{r}(R)\right|\right\rangle=\left\langle\left|\frac{\mathbf{w}(R) \cdot \mathbf{r}}{r}\right|\right\rangle,
$$

where $\mathbf{r}$ is the vector connecting the centers of the collision pair, $r=|\mathbf{r}|$, and $\mathbf{w}(R)=\mathbf{v}_{p 1}-\mathbf{v}_{p 2}$ is the relative velocity between the two particles. The angle brackets denote an ensemble average over all collision pairs.

In order to study the effects of different filter widths on particle collision-related statistics, we calculate $\langle g(R)\rangle$, $\left\langle\left|w_{r}(R)\right|\right\rangle$, and $\left\langle\dot{N}_{c}\right\rangle$ of particles with different Stokes numbers $\mathrm{St}_{K}=0.5,1$, and 5 in different FDNS flow fields with the cutoff wavenumbers $k_{\mathrm{cf}}=64, k_{\mathrm{cf}}=42$, and $k_{\mathrm{cf}}=21$, respectively. The statistics of the energy, enstrophy, and energy dissipation rate in $256^{3}$ DNS, FDNS, and $64^{3}$ LES flow fields are given in Table II. As the energy spectrum of FDNS for $k \leq k_{\mathrm{cf}}$ is the same as that of the DNS, typically very little turbulent kinetic energy is contained within high wavenumbers $\left(k>k_{\mathrm{cf}}\right)$. The percentage of energy filtered out in the FDNS is negligible except that the cutoff wavenumber is sufficiently low. For the largest filter width at $k_{\mathrm{cf}}=21$ in the present study, only $3 \%$ of the kinetic energy is filtered out, so the rms turbulent velocity in the FDNS flow field differs very little from that of the DNS flow field. However, as enstrophy is defined as $\int_{k_{0}}^{k_{\mathrm{cf}}} 2 k^{2} E(k) d k$, a large portion of enstrophy is distributed at high wavenumbers, thus a large percentage of enstrophy is filtered out in FDNS. For the case of $k_{\mathrm{cf}}=21$, about $45.7 \%$ of enstrophy is filtered out. With the same spatial resolution in the FDNS $\left(k_{\mathrm{cf}}=21\right)$ and $64^{3} \mathrm{LES}$, the total turbulent energy of LES is lower than that of FDNS, and the enstrophy is even lower due to the overdissipation of the eddy viscosity SGS model. The relative errors in $\langle g(R)\rangle$, $\left\langle\left|w_{r}(R)\right|\right\rangle$, and $\left\langle\dot{N}_{c}\right\rangle$, using DNS results as a benchmark, are shown in Fig. 8. For a given Stokes number, the relative errors of these statistics increase with increasing filter width, and the relative errors for $\langle g(R)\rangle,\left\langle\left|w_{r}(R)\right|\right\rangle$, and $\left\langle\dot{N}_{c}\right\rangle$ are not the same. For example, when $k_{\mathrm{cf}}=21$ and $\mathrm{St}_{K}=0.5$, the relative errors for $\langle g(R)\rangle,\left\langle\left|w_{r}(R)\right|\right\rangle$, and $\left\langle\dot{N}_{c}\right\rangle$ are $-0.37,-0.29$, and -0.57 , respectively. For a given filter width, the filter operation has different effects on the motion of particles with 
TABLE II. Kinetic energy, enstrophy in DNS $\left(256^{3}\right)$, FDNS, and LES $\left(64^{3}\right)$.

\begin{tabular}{lccccc}
\hline \hline Method & DNS $\left(256^{3}\right)$ & $k_{\mathrm{cf}}=64$ & $k_{\mathrm{cf}}=42$ & $k_{\mathrm{cf}}=21$ & LES $\left(64^{3}\right)$ \\
\hline$k_{\mathrm{cf}} \eta$ & 1.15 & 0.864 & 0.567 & 0.285 & $\ldots$ \\
Energy & 561.3 & 561.0 & 559.5 & 544.2 & 514.5 \\
Enstrophy & 77290.6 & 74409.8 & 66833.7 & 41994.6 & 33330.6 \\
Actual dissipation rate & 3771.4 & 3631 & 3261 & 2049 & 1626.6 \\
Energy filtered out $(\%)$ & $\ldots$ & 0.05 & 0.32 & 3.04 & 8.3 \\
Enstrophy filtered out $(\%)$ & $\ldots$ & 3.72 & 13.5 & 45.7 & 56.9 \\
\hline \hline
\end{tabular}

different Stokes numbers. The reason is that particles with different inertia are related to different length scales of eddies. ${ }^{34}$ It implies that filtering operation can result in a higher or lower prediction of $\langle g(R)\rangle$ for different Stokes numbers and always lower prediction of $\left\langle\left|w_{r}(R)\right|\right\rangle$ and $\left\langle\dot{N}_{c}\right\rangle$. It is important to note that, for small Stokes numbers, the relative error on the relative velocity is much larger than the relative amount of turbulent kinetic energy removed, since the main contribution to the particle relative motion comes from small-scale fluid motion. Our simulation results about the effects of filtering on particle collision rate are consistent with those of Fede and Simonin. ${ }^{14}$ We have included the data in Fig. 28 of Fede and Simonin ${ }^{14}$ in Fig. 8(c) for comparison. Some of the quantitative difference between their data and ours could be due to the fact that Fede and Simonin ${ }^{14}$ assumed zero settling and the Stokes numbers are not the same. The effects of inertia on particle-pair statistics will be discussed in detail later.

\section{E. Effects of eddy viscosity SGS model on collision-related statistics}

A spectral eddy viscosity SGS model [see Eqs. (6) and (7)] is used to close the filtered Navier-Stokes equations in LES. From Fig. 1, one can see that the spectral eddy viscosity SGS model overdissipates the turbulent kinetic energy, especially near the cutoff wavenumbers, making the vorticity field in LES more coherent than that in FDNS with the same spatial resolution. As particle turbulent collision rate is related to the particle preferential concentration and the averaged radial relative velocity, we will first study the influences of SGS motions and the spectral eddy viscosity SGS model on the two aforementioned quantities and then the collision rates using both a priori and a posteriori tests, i.e., we will compare the results from the DNS and the ones from the FDNS and the LES flow fields, respectively. This study will be carried out over a wide range of the Stokes numbers.
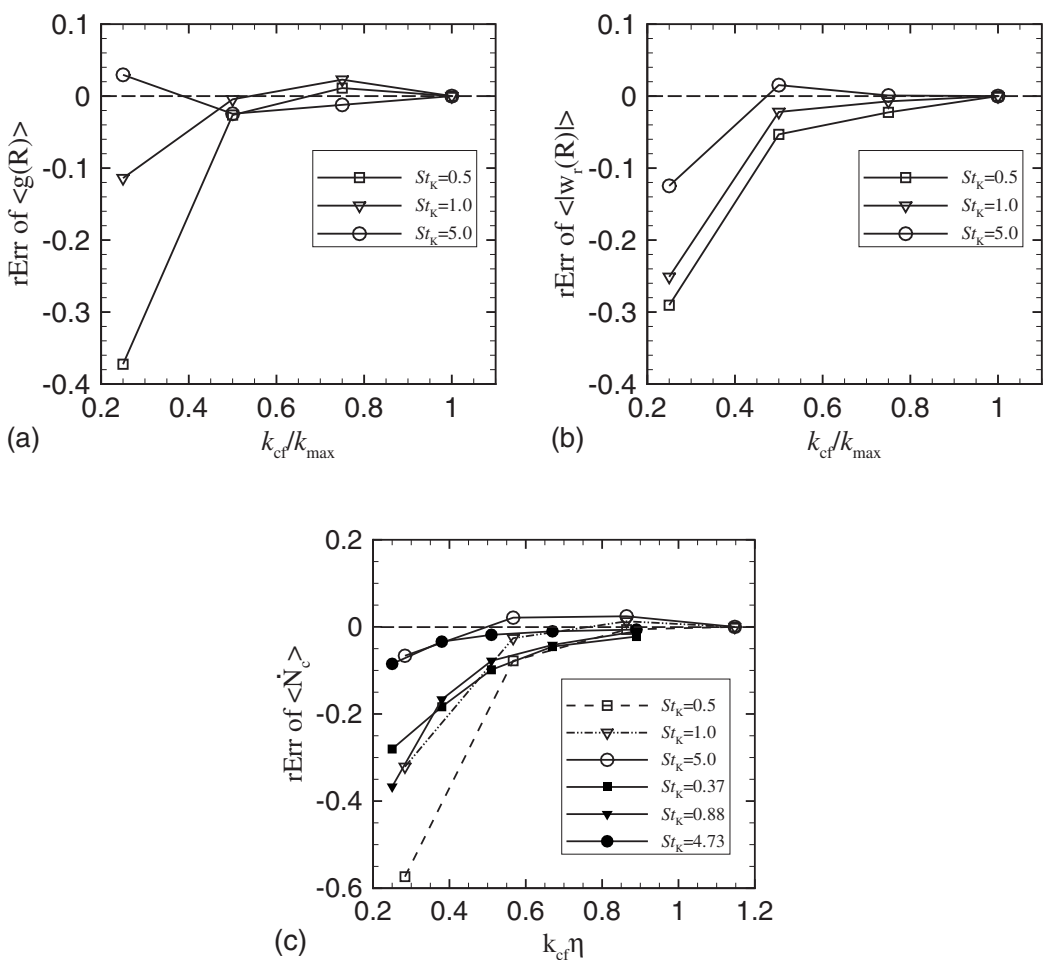

FIG. 8. Effects of filter width on the collision-related statistics of different Stokes numbers, where rErr denotes the relative error of the result from FDNS to that from DNS, $\mathrm{rErr}=\left(\alpha_{\mathrm{FDNS}}-\alpha_{\mathrm{DNS}}\right) / \alpha_{\mathrm{DNS}}$, and $\alpha$ represents a statistical quantity and the open symbols in (c) are our numerical results and the solid symbols are obtained from Fig. 28 of Fede and Simonin (Ref. 14). (a) The radial distribution function at contact $\langle g(R)\rangle$; (b) the radial relative velocity at contact $\left\langle\left|w_{r}(R)\right|\right\rangle$; (c) the particle collision rate $\left\langle\dot{N}_{c}\right\rangle$. 


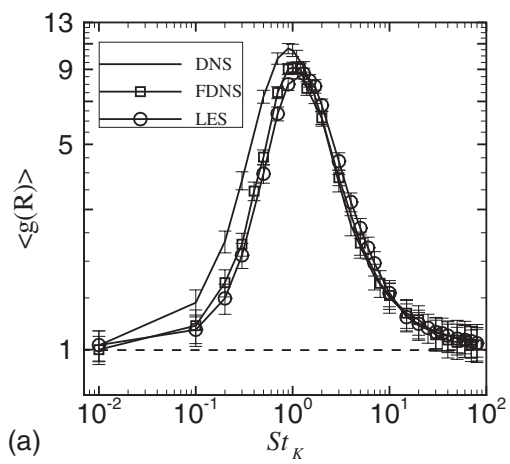

(a)

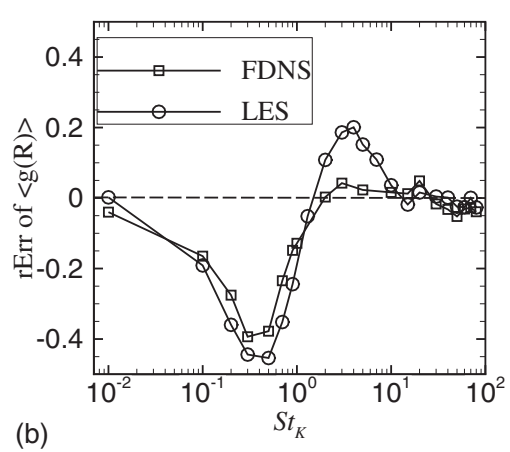

FIG. 9. Effects of filtering operation and spectral eddy viscosity model on $\langle g(R)\rangle$ of different Stokes numbers in LES $\left(64^{3}\right)$, FDNS $\left(k_{\mathrm{cf}}=21\right)$, and DNS $\left(256^{3}\right)$ flow fields. (a) $\langle g(R)\rangle$ variation with $\mathrm{St}_{k}$; (b) relative error of $\langle g(R)\rangle$ from LES and FDNS to the DNS results.

\section{Radial distribution function}

A nonuniform distribution of particles (the accumulation effect) at the Stokes numbers near one is mainly induced by small-scale eddies which are directly related to the cutoff wavenumber in FDNS and related to both the cutoff wavenumber and the spectral eddy viscosity SGS model in LES. For $k_{\mathrm{cf}}=21$, the space resolution in $256^{3} \mathrm{DNS}$ is four times of the ones in FDNS and LES $\left(\Delta x_{\mathrm{LES}}=\Delta x_{\mathrm{FDNS}}=4 \Delta x_{\mathrm{DNS}}\right)$. Figure 9(a) shows the dependence of $\langle g(R)\rangle$ from the DNS, FDNS, and LES on the Stokes numbers, and Fig. 9(b) plots the relative errors (rErr) of the FDNS and LES to the DNS results. Figure 9 shows that there are three regimes for $\langle g(R)\rangle$. In the limit cases of both $\mathrm{St}_{K} \rightarrow 0$ and $\mathrm{St}_{K} \rightarrow \infty$, particles tend to be uniformly distributed and $\langle g(R)\rangle \rightarrow 1$ since particles with a very small Stokes number are distributed like fluid elements and particles with a very large Stokes number are not responsive to flow structures. For the intermediate Stokes numbers, particles interact with a range of fluid eddies and tend to accumulate in the regions of low vorticities, leading to the large values of $\langle g(R)\rangle$ with a peak value around $\mathrm{St}_{K}=1$ in the DNS. This trend is consistent with the previous observations. ${ }^{18,33}$

Our results from Figs. 8(a) and 9 are qualitatively similar to Fig. 22 of Fede and Simonin, ${ }^{14}$ where a different measure of preferential concentration was used. For very small Stokes numbers, $\mathrm{St}_{K}<1$, the effect of filtering is to decrease the particle accumulation as small-scale eddies most relevant to accumulation have been removed. A very interesting observation from Fig. 9 is that the magnitude of the relative error in $\langle g(R)\rangle$ for the FDNS flow has its maximum near $\mathrm{St}_{K}$ $=0.5$, with a maximum reduction of as much as $40 \%$. For $\mathrm{St}_{K}>0.5$, the magnitude of the relative error decreases with increasing the Stokes number, indicating that the SGS eddies make less and less contribution to the preferential concentration. For $\mathrm{St}_{K}>1$, the FDNS yields a slightly higher $\langle g(R)\rangle$ than the DNS result, indicating that the accumulation of these particles is more controlled by resolved eddies and the SGS eddies may act to randomize the particle distribution. Therefore, filtering out the random small eddies increases $\langle g(R)\rangle$. For $\mathrm{St}_{K}>10$, the particles are not responsive to the SGS eddies so the FDNS yields accurate results.

Compared with the FDNS at the same spatial resolution, the LES predicts an even lower value of $\langle g(R)\rangle$ for small Stokes numbers $\mathrm{St}_{K}<1$. This is due to the overdissipation of the SGS model which tends to damp the eddies near the
SGS, making them contribute less to preferential concentration. Interestingly, for the intermediate Stokes numbers 1 $<\mathrm{St}_{K}<10$, a higher $\langle g(R)\rangle$ is realized when compared to the FDNS. This results from the fact that the resolved large-scale eddies are more coherent in space and time, particles have a longer interaction time with low vorticity regions, leading to a somewhat stronger preferential concentration. For the particles with very large Stokes numbers, $\mathrm{St}_{K}>10$, the results from the DNS, FDNS, and LES are similar.

The peak values of $\langle g(R)\rangle$ in the FDNS and LES occur at a slightly larger Stokes numbers than that in the DNS. This is expected as the effective time scales for strongest vortical structures in the FDNS and LES are larger than $\tau_{K}$.

\section{The radial relative velocity}

For particles with large Stokes numbers, the velocities of the collision pair are uncorrelated and the relative velocity at contact is mainly determined by the energy-contained eddies. ${ }^{18}$ The energy-contained eddies are supposed to be at large scales and well resolved by LES. However, in the limit when particle inertia goes to zero, the relative velocity of particles is essentially determined by the local gradient of fluid velocity field provided that the collision radius $R$ is small. In this limit, Saffman and Turner ${ }^{35}$ provided a prediction for $\left\langle\left|w_{r}(R)\right|\right\rangle$ in terms of the turbulent kinetic energy dissipation rate or the enstrophy of the flow field. As shown in Table II, although the relative percentage of turbulent kinetic energy that is filtered out in LES is negligible, a significant amount of enstrophy has been filtered out. Thus, we expect that a large error could arise in the LES prediction of the relative velocities for particles at very small Stokes numbers.

Figure 10(a) shows the dependence of $\left\langle\left|w_{r}(R)\right|\right\rangle$ on the Stokes number, and Fig. 10(b) plots the relative errors of the FDNS and LES to the DNS results. It can be seen that $\left\langle\left|w_{r}(R)\right|\right\rangle$ is small when the particle Stokes number is small $\left(\mathrm{St}_{K}<1\right)$. The reason for this behavior is that, in this limit, the two particles are strongly correlated as they are transported by large eddies, and only small-scale eddies are effective in generating particle relative motion. It is also observed that the quantity $\left\langle\left|w_{r}(R)\right|\right\rangle$ increases rapidly with the particle Stokes number for $\mathrm{St}_{K}<20$. As the particle Stokes number increases, the large-scale turbulent fluctuations become effective in generating relative motion as different eddies encountered by the particles during their passage to a geometric 


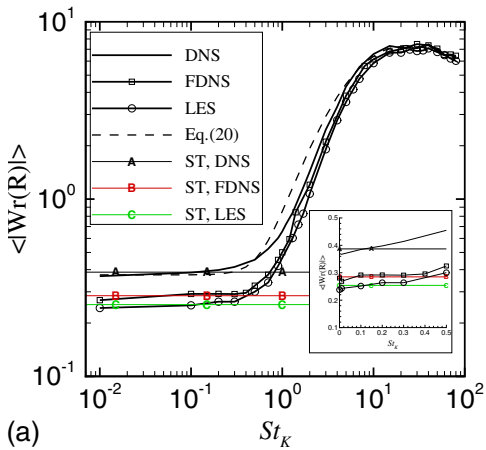

(a)

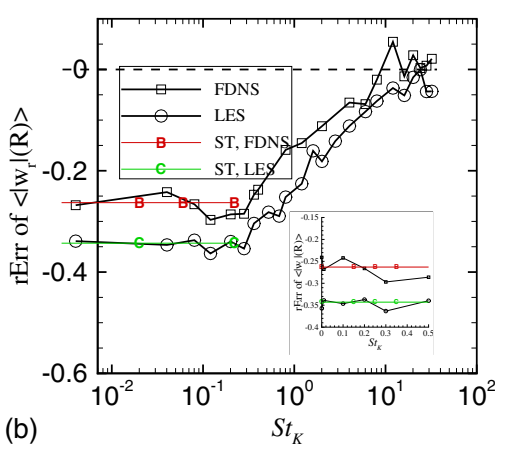

FIG. 10. (Color online) Effects of filtering operation and spectral eddy viscosity model on $\left\langle\left|w_{r}(R)\right|\right\rangle$ for particles at different Stokes numbers in LES $\left(64^{3}\right)$, FDNS $\left(k_{\mathrm{cf}}=21\right)$, and DNS $\left(256^{3}\right)$ flow fields. $\theta=1.8 \tau_{p} / T_{E}$, $C_{w}=1.5$ are used to fit the numerical results in Eq. (21). ST in the legends denotes the ST theoretical prediction at zero Stokes number. (a) $\left\langle\left|w_{r}(R)\right|\right\rangle$ variation with $\mathrm{St}_{k}$; (b) relative error in $\left\langle\left|w_{r}(R)\right|\right\rangle$ of the LES and FDNS results using the DNS results as the benchmark. contact can all contribute to the relative motion (i.e., the memory effect). For $\mathrm{St}_{K}>20$, the relative velocity begins to decrease slowly with the Stokes number, as there are no more large-scale fluctuations in the flow to disturb the particle motion. ${ }^{18}$ One can observe that the relative velocity in the FDNS is smaller than that in the DNS, but larger than that in the LES. Therefore, the relative error in the LES is larger than that in the FDNS for particles with small and intermediate Stokes numbers. As particle inertia increases, the radial relative velocity is affected more and more by the large-scale turbulence. The relative errors for FDNS and LES decrease with increasing particle Stokes number.

We shall now analyze the various behaviors of the radial relative velocity with the existing theories. The radial relative velocity for finite Stokes number particles is governed by the interaction of particles with both large-scale energetic eddies and small-scale dissipation eddies. By assuming that both PDFs of $w_{r}$ and fluid velocity gradient $\partial u / \partial x$ are Gaussian, Wang et al. ${ }^{18}$ developed a model for $\left\langle\left|w_{r}(R)\right|\right\rangle$ which combines the turbulent acceleration mechanism of the largescale turbulent motions and shear mechanism of the local small-scale dissipative motions as

$$
\left\langle\left|w_{r}(R)\right|\right\rangle=\sqrt{\frac{2}{\pi}}\left\langle w_{r}^{2}(R)\right\rangle=\sqrt{\frac{2}{\pi}} \sqrt{\left\langle w_{r, \text { shear }}^{2}\right\rangle+\left\langle w_{r, \text { accel }}^{2}\right\rangle} .
$$

The acceleration mechanism ${ }^{36}$ was expressed as

$$
\begin{aligned}
\frac{\left\langle w_{r, \text { accel }}^{2}\right\rangle}{u^{\prime 2}}= & C_{w} \frac{2 \gamma \theta}{\gamma-1}\left[1-\frac{(1+2 \theta)^{1 / 2}}{1+\theta}\right] \\
& \times\left[\frac{1}{(1+\theta)^{2}}-\frac{1}{(1+\gamma \theta)^{2}}\right],
\end{aligned}
$$

where $\theta=2.5 \tau_{p} / T_{E}, \gamma=0.183 u^{\prime 2} / v_{K}^{2}, T_{E}$ is the Eulerian integral time scale, $v_{K}$ is the Kolmogorov velocity, and the factor $C_{w}$ is used to fit the numerical results. Equation (21) shows that the effect of acceleration mechanism on radial relative velocity depends on particle relative inertia $\theta$, turbulent intensity $u^{\prime 2}$, and the turbulent Reynolds number $\operatorname{Re}_{\lambda}$ since $u^{\prime 2} / v_{K}^{2}=\operatorname{Re}_{\lambda} / \sqrt{15}$. For particles with very small Stokes numbers, the relative velocity is related to the local shear rate of the flow ${ }^{35}$

$$
\frac{\left\langle w_{r, \text { shear }}^{2}\right\rangle}{v_{K}^{2}}=\frac{1}{15}\left(\frac{R}{\eta}\right)^{2},
$$

where $R$ is the collision radius and was set to $0.5 \eta$ in the simulation. Thus, the shear contribution scales with the small-scale flow velocity $v_{K}$. The predictions of $\left\langle\left|w_{r}\right|\right\rangle$ using Eq. (20) are compared with the DNS results in Fig. 10(a). It is shown that Eq. (20) can provide a reasonable prediction of the simulated $\left\langle\left|w_{r}\right|\right\rangle$ data.

It is noted that Eq. (20) reduces to the Saffman and Turner's (ST) theory for particle with zero inertia or $\theta \rightarrow 0$, as

$$
\left\langle\left|w_{r}(R)\right|\right\rangle=\sqrt{\frac{2}{15 \pi}} R \sqrt{\frac{\varepsilon}{\nu}},
$$

then $\left\langle\left|w_{r}(R)\right|\right\rangle$ is directly proportional to the square root of the dissipation rate in DNS, FDNS, and LES flow fields when $R$ and $\nu$ are prescribed. The ST predictions of $\left\langle\left|w_{r}(R)\right|\right\rangle$ are $0.387,0.285$, and 0.254 for DNS, FDNS, and LES flow fields, respectively, and these are shown in Fig. 10(a) as well. The molecular viscosity coefficient used for the LES case is the same as the DNS case, $\nu=0.0488$. Indeed, for particles with very small Stokes numbers, the data agree with the ST predictions. This is true even for FDNS and LES when the actual dissipation rates (see Table II) and molecular viscosity are used in the ST theory. The inset in Fig. 10(a) is an enlargement for the small $\mathrm{St}_{K}$ region using linear scales, where additional data corresponding to fluid particles are added. The average radial relative velocity for fluid particles was calculated from the Eulerian velocity field using

$$
\begin{aligned}
\left\langle\left|w_{r}(R)\right|\right\rangle= & \frac{1}{3}[\langle|u(x, y, z, t)-u(x+R, y, z, t)|\rangle \\
& +\langle|v(x, y, z, t)-v(x, y+R, z, t)|\rangle \\
& +\langle|w(x, y, z, t)-w(x, y, z+R, t)|\rangle],
\end{aligned}
$$

where $u, v$, and $w$ are the velocity along the $x, y$, and $z$ axes, respectively. The numerical results of $\left\langle\left|w_{r}(R)\right|\right\rangle$ between fluid particles are $0.37,0.281$, and 0.238 for DNS, FDNS, and LES flow fields, respectively. Due to the loss of large amount of enstrophy in FDNS and LES flow fields, FDNS and LES both underpredict the relative velocities when compared to DNS for $\mathrm{St}_{K}=0$. Furthermore, the relative reductions in FDNS and LES in this limit are different due to different levels of loss of enstrophy. The very minor discrepancies between our numerical results and the ST theory might come 

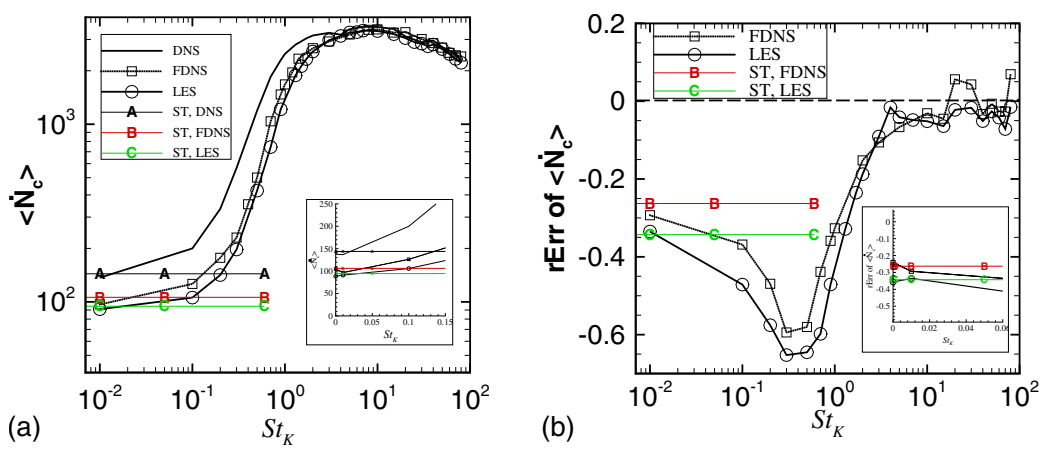

FIG. 11. (Color online) Effects of filtering operation and spectral eddy viscosity model on $\left\langle\dot{N}_{c}\right\rangle$ for particles with different Stokes numbers in LES $\left(64^{3}\right)$, FDNS $\left(k_{\mathrm{cf}}=21\right)$, and DNS $\left(256^{3}\right)$ flow fields. ST in the legends denotes the Saffman and Turner's theory for small Stokes number particles. (a) $\left\langle\dot{N}_{c}\right\rangle$ variation with $\mathrm{St}_{k}$; (b) relative error of $\left\langle\dot{N}_{c}\right\rangle$ from the LES and FDNS to the DNS results. from the finite particle diameter of $0.5 \eta$ assumed in the simulations since the ST theory assumes $R \ll \eta$.

At the high Stokes number limit, one can observe in Fig. 10 that $\left\langle\left|w_{r}(R)\right|\right\rangle$ from DNS, FDNS, and LES are in good agreement and the relative errors of FDNS and LES approach zero. Since the velocities of the two colliding particles in this limit are completely uncorrelated and can be characterized by a Maxwellian distribution, the relative velocity is also Maxwellian. According to the analysis based on the kinetic theory for the very high Stokes number limit $\left(\mathrm{St}_{k} \rightarrow \infty\right),\left\langle\left|w_{r}(R)\right|\right\rangle$ can be expressed as ${ }^{33,37}$

$$
\left\langle\left|w_{r}(R)\right|\right\rangle=\frac{2}{\sqrt{\pi}} \sqrt{\frac{1}{3} \bar{v}_{p}^{2}},
$$

where $\overline{v_{p}^{2}}$ is the variance of particle velocity. Since $\left\langle\left|w_{r}(R)\right|\right\rangle$ is now related to the one-point statistics $\overline{v_{p}^{2}}$, which is almost unaffected by the small-scale fluid motion for particles with high Stokes numbers. ${ }^{14,38}$ Thus, the agreement of FDNS and LES with DNS at the high Stokes number limit shown in Fig. 10 are qualitatively consistent with this theoretical argument. A quantitative comparison in this limit will be provided in Sec. III E 3 when results on the collision rate are discussed.

\section{Particle collision rate}

Figure 11 shows the dynamically detected collision rate of particles from the DNS, FDNS, and LES. Again, the ST predictions are plotted for the small inertia limit using the dissipation rates in DNS, FDNS, and LES, respectively. The general trends from the three flow fields shown in Fig. 11(a) are similar. The collision rate increases very rapidly for small Stokes numbers, reaches a peak at $\mathrm{St}_{K}=10$, and then drops slowly with an increase in $\mathrm{St}_{K}$. This behavior is consistent with the results for the collision kernel found by Sundaram and Collins ${ }^{33}$ and Zhou et al. ${ }^{39}$ The peak reflects both the effects of particle interaction with small and large scales of fluid motions. The interaction of heavy particle with smallscale motions produces a nonuniform particle distribution which enhances the collision rate through a large value of the radial distribution function at contact $\langle g(R)\rangle$. On the other hand, as Stokes number increases, the turbulent transport effect which produces a large value of relative velocity, $\left\langle\left|w_{r}(R)\right|\right\rangle$, will dominate the collision rates. ${ }^{18}$ For small Stokes numbers, filtering causes a lower $\langle g(R)\rangle$ and $\left\langle\left|w_{r}(R)\right|\right\rangle$, as shown in Figs. 9 and 10, and thus a lower par- ticle collision rate according to Eq. (18). The maximum relative errors occur at $\mathrm{St}_{K}=0.5$ and are larger than $60 \%$. For intermediate Stokes numbers, $1<\mathrm{St}_{K}<10$, however, filtering causes an overestimation of $\langle g(R)\rangle$ but an underestimation of $\left\langle\left|w_{r}(R)\right|\right\rangle$. This is a fortunate combination and leads to a rather accurate prediction of the overall collision rate. In fact, for $\mathrm{St}_{K}>3$, both FDNS and LES yield very reasonable results for collision rate, and the difference between FDNS and LES is not noticeable. The nonmonotonic variation of the relative errors in $\left\langle\dot{N}_{c}\right\rangle$ for intermediate Stokes numbers shown in Fig. 11(b) is a combination of the effects of $\langle g(R)\rangle$ and $\left\langle\left|w_{r}(R)\right|\right\rangle$.

In the two limiting cases of very small and very high Stokes numbers, particles uniformly distribute in the flow field, $\langle g(R)\rangle \approx 1.0$, the collision rate $\left\langle\dot{N}_{c}\right\rangle$ depends only on $\left\langle\left|w_{r}(R)\right|\right\rangle$. Based on Eqs. (18) and (23), the average collision rate for fluid particles (the zero inertia limit) is

$$
\left\langle\dot{N}_{c}\right\rangle=\sqrt{\frac{8 \pi}{15}} R^{3} \frac{n_{0}^{2}}{2} \sqrt{\frac{\varepsilon}{\nu}},
$$

where the average number density used in the simulations was $n_{0}=400000 /(2 \pi)^{3} \approx 1613$. Figure 11 shows that both FDNS and LES underpredict the collision rates due to the losses of dissipation rate in FDNS and LES flow fields, as implied by Eq. (26).

Based on Eqs. (18) and (25), the average collision rate for particles with very high Stokes numbers $\left(\operatorname{St}_{k} \rightarrow \infty\right)$ is

$$
\left\langle\dot{N}_{c}\right\rangle=2 \sqrt{\pi} n_{0}^{2} R^{2} \sqrt{\frac{1}{3} \overline{v_{p}^{2}}} .
$$

The quantitative comparison between the numerical collision rates and the theoretical prediction using Eq. (27) for particles with high Stokes numbers is shown in Fig. 12, where the collision rate is normalized by $\tilde{N}_{c}=\left\langle\dot{N}_{c}\right\rangle /\left(\sqrt{\overline{v_{p}^{2}} / 3} R^{2} n_{0}^{2} / 2\right)$, $\overline{v_{p}^{2}}$ is obtained from the simulated particle velocities in DNS, FDNS, and LES flow fields, respectively. The theoretical value of $\tilde{N}_{c}$ for high Stokes number limit is $\tilde{N}_{c}=4 \sqrt{\pi}=7.09$ based on Eq. (27). From Fig. 12, we observe that there is a good agreement in the collision rates obtained from DNS, FDNS, and LES. However, the theoretical value at the high Stokes number limit $\left(\mathrm{St}_{K} \rightarrow \infty\right)$ is still significantly above the values seen in the simulations. We note that $\mathrm{St}_{K}=80$ for the DNS flow case is equivalent to $\tau_{p} / T_{E} \approx 5.7$, where $T_{E}$ is the Eulerian integral time. Therefore, the theoretical limit would require a much larger $\tau_{p}$ than those shown in Fig. 12. The 


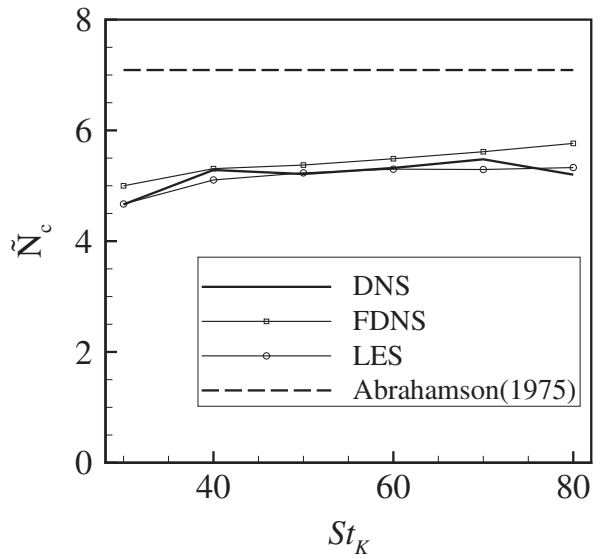

FIG. 12. The comparison between the normalized numerical collision rates with the kinetic theory of Abrahamson (Ref. 37) for particles with high Stokes numbers.

difficulty to reproduce the kinetic high Stokes number limit in DNS was noted in previous studies (for example, see Fig. 4 in Ref. 33 and Fig. 6 in Ref. 40).

\section{CONCLUSIONS}

The turbulent collision of heavy particles is a small-scale process, where the small-scale velocity field plays an important role. This represents a challenge to LES of such a process. In LES, the large-scale velocity is explicitly obtained by solving the filtered Navier-Stokes equations which are closed with an eddy viscosity SGS model such as Eq. (6) to account for the effects of the SGS velocity on the large-scale one, while the SGS velocity itself cannot be obtained explicitly. The particle-pair and collision-related statistics of heavy particles are significantly affected by the SGS velocity fields. We have demonstrated here that the errors in LES of turbulent collision can be attributed to two sources: the absence of SGS velocity due to filtering and the error from the eddy viscosity SGS model. We have attempted to clearly quantify the errors from these two sources using both $a$ priori and $a$ posteriori tests.

We carefully examine the relative errors on the radial distribution functions at contact and the radial relative velocities at contact caused by filtering and the SGS model. The relative errors of collision-related statistics increase when the filter width increases, and for particles with small Stokes numbers, the relative errors are much larger than the relative amount of turbulent kinetic energy filtered out. For a given filter width, $k_{\mathrm{cf}}=21$ or $\eta k_{\mathrm{cf}}=0.284$, these relative errors are found to be quite significant for a wide range of particle Stokes numbers, as a result of the interactions of particles with small-scale fluid motions. The errors exhibit nonmonotonic dependencies on the particle Stokes numbers. For a range of intermediate Stokes numbers, such as $1<\mathrm{St}_{K}<10$, the radial distribution functions at contact in the FDNS and LES can be larger than that in the DNS. This nonmonotonic variations have a fortunate consequence: the collision rate can be well predicted by the FDNS and LES when $\mathrm{St}_{K}>3$ since the overestimation of $\langle g(R)\rangle$ partly cancels the underestimation of $\left\langle\left|w_{r}(R)\right|\right\rangle$. For $\mathrm{St}_{K}<1$, we find that both FDNS and LES with the spectral eddy viscosity SGS model tend to underpredict the radial distribution function at contact and radial relative velocity at contact. As a result, the error on the turbulent collision rate can become quite larger. For example, at $\mathrm{St}_{K}=0.5$, the relative error on the collision rate can be as large as $60 \%$ in LES.

By examining the spectral content of the particle concentration field in DNS, we have demonstrated that the dominant scales governing the preferential concentration change with the Stokes number. Those observations further clarify the dynamics of heavy particles relevant to the SGS motions in LES. Our analysis suggests that, for $\mathrm{St}_{K}<3$, a particle SGS model must include the effects of the SGS motions on the turbulent collision of heavy particles.

\section{ACKNOWLEDGMENTS}

This work was supported by CAS (Grant No. KJCX2SW-L08), 973 Program of China (Grant No. 2007CB8 14800), NSFC (Grant Nos. 10628206, 10732090, and 10702074), the LNM initial funding for young investigators, and SRF for ROCS, SEM. L.-P.W. acknowledges support by the U.S. National Science Foundation (under Grant Nos. ATM-0527140 and ATM-0730766) during the course of this study and by NSFC (Grant No. 10628206) which made it possible for him to visit and accomplish this work at LNM, Institute of Mechanics, Chinese Academy of Sciences in China. The constructive comments from two anonymous reviewers are greatly appreciated.

${ }^{1}$ C. T. Crowe, M. Sommerfeld, and Y. Tsuji, Multiphase Flows with Droplets and Particles (CRC, Boca Raton, FL, 1998).

${ }^{2}$ C. T. Crowe, Multiphase Flow Handbook (CRC, Boca Raton, FL, 2006). ${ }^{3}$ P. A. Vaillancourt and M. K. Yau, "Review of particle-turbulence interactions and consequences for cloud physics," Bull. Am. Meteorol. Soc. 81, 285 (2000)

${ }^{4}$ M. G. Pai and S. Subramaniam, "Modeling interphase turbulent kinetic energy transfer in Lagrangian-Eulerian spray computations," Atomization Sprays 16, 807 (2006).

${ }^{5}$ L.-P. Wang, B. Rosa, H. Gao, G.-W. He, and G. D. Jin, "Turbulent collision of inertial particles: Point-particle based, hybrid simulations and beyond," Int. J. Multiphase Flow 35, 854 (2009).

${ }^{6}$ S. C. Kassinos, C. A. Langer, G. Iaccarino, and P. Moin, Complex Effects in Large Eddy Simulations (Springer, Berlin, 2007).

${ }^{7}$ F. Yeh and U. Lei, "On the motion of small particles in a homogeneous isotropic turbulent flow," Phys. Fluids A 3, 2571 (1991).

${ }^{8}$ Q. Wang and K. D. Squires, "Large eddy simulation of particle-laden turbulent channel flow," Phys. Fluids 8, 1207 (1996).

${ }^{9}$ V. Armenio, U. Piomelli, and V. Fiorotto, "Effect of the subgrid scales on particle motion," Phys. Fluids 11, 3030 (1999).

${ }^{10}$ Y. Yamamoto, M. Potthoff, T. Tanaka, T. Kajishima, and Y. Tsuji, "Largeeddy simulation of turbulent gas-particle flow in a vertical channel: Effect of considering inter-particle collisions," J. Fluid Mech. 442, 303 (2001).

${ }^{11}$ J. G. M. Kuerten, "Subgrid modeling in particle-laden channel flow," Phys. Fluids 18, 025108 (2006).

${ }^{12}$ B. Shotorban and F. Mashayek, "Modeling subgrid-scale effect on particles by approximate deconvolution," Phys. Fluids 17, 081701 (2005).

${ }^{13}$ B. Shotorban and F. Mashayek, "A stochastic model for particle motion in large-eddy simulation,” J. Turbul. 7, 18 (2006).

${ }^{14} \mathrm{P}$. Fede and O. Simonin, "Numerical study of the subgrid fluid turbulence 
effects on the statistics of heavy colliding particles," Phys. Fluids 18, 045103 (2006)

${ }^{15}$ A. S. Berrouk, D. Laurence, J. J. Riley, and D. E. Stock, "Stochastic modelling of inertial particle dispersion by subgrid motion for LES of high Reynolds number pipe flow," J. Turbul. 8, 50 (2007).

${ }^{16} \mathrm{M}$. Bini and W. P. Jones, "Large-eddy simulation of particle-laden turbulent flows," J. Fluid Mech. 614, 207 (2008).

${ }^{17}$ J. Pozorski and S. V. Apte, "Filtered particle tracking in isotropic turbulence and stochastic modeling of subgrid-scale dispersion," Int. J. Multiphase Flow 35, 118 (2009).

${ }^{18}$ L.-P. Wang, A. S. Wexler, and Y. Zhou, "Statistical mechanical description and modelling of turbulent collision of inertia particles," J. Fluid Mech. 415, 117 (2000).

${ }^{19}$ L. I. Zaichik and V. M. Alipchenkov, "Pair dispersion and preferential concentration of particles in isotropic turbulence," Phys. Fluids 15, 1776 (2003).

${ }^{20}$ A. M. Wood, W. Hwang, and J. K. Eaton, "Preferential concentration of particles in homogeneous and isotropic turbulence," Int. J. Multiphase Flow 31, 1220 (2005).

${ }^{21}$ J.-P. Chollet and M. Lesieur, "Parameterization of small scales of threedimensional isotropic turbulence utilizing spectral closure," J. Atmos. Sci. 38, 2747 (1981)

${ }^{22}$ J.-P. Chollet, in Turbulent Shear Flows 4, edited by L. J. S. Bradbury, F. Durst, and B. E. Launder (Springer, Berlin, 1983), pp. 62-72.

${ }^{23}$ G.-W. He, R. Rubinstein, and L.-P. Wang, "Effects of subgrid-scale modeling on time correlations in large eddy simulation," Phys. Fluids 14, 2186 (2002).

${ }^{24}$ P. Fede, O. Simonin, P. Villedieu, and K. D. Squires, "Stochastic modeling of turbulent subgrid fluid velocity along inertia particle trajectories," Proceedings of the 2006 CTR Summer Program (Center for Turbulence Research, Stanford, CA, 2006).

${ }^{25}$ E. Eswaran and S. B. Pope, "An examination of forcing in direct numerical simulations of turbulence," Comput. Fluids 16, 257 (1988).

${ }^{26}$ L.-P. Wang and M. R. Maxey, "Settling velocity and concentration distribution of heavy particles in homogeneous isotropic turbulence," J. Fluid Mech. 256, 27 (1993).
${ }^{27}$ Y. Yang, G.-W. He, and L.-P. Wang, "Effects of subgrid-scale modeling on Lagrangian statistics in large-eddy simulation," J. Turbul. 9, 8 (2008).

${ }^{28}$ M. R. Maxey and J. J. Riley, "Equation of motion for a small rigid sphere in a nonuniform flow," Phys. Fluids 26, 883 (1983).

${ }^{29}$ W. C. Reade and L. R. Collins, "Effect of preferential concentration on turbulent collision rates," Phys. Fluids 12, 2530 (2000).

${ }^{30}$ O. Ayala, B. Rosa, L.-P. Wang, and W. W. Grabowski, "Effects of turbulence on the geometric collision rate of sedimenting droplets: Part I. Results from direct numerical simulation," New J. Phys. 10, 075015 (2008) (focus issue on cloud physics).

${ }^{31}$ M. P. Allen and D. J. Tildesley, Computer Simulation of Liquids (Oxford University Press, New York, 1987).

${ }^{32}$ M. R. Maxey, "The gravitational settling of settling of aerosol particles in homogeneous turbulence and random flow fields," J. Fluid Mech. 174, 441 (1987).

${ }^{33}$ S. Sundaram and L. Collins, "Collision statistics in an isotropic particleladen turbulent suspension. Part I: Direct numerical simulations," J. Fluid Mech. 335, 75 (1997).

${ }^{34} \mathrm{H}$. Yoshimoto and S. Goto, "Self-similar clustering of inertial particles in homogeneous turbulence," J. Fluid Mech. 577, 275 (2007).

${ }^{35}$ P. G. Saffman and J. S. Turner, "On the collision of drops in turbulent clouds," J. Fluid Mech. 1, 16 (1956); "Corrigendum," ibid. 196, 599(E) (1988).

${ }^{36}$ F. E. Kruis and K. A. Kusters, "The collision rate of particles in turbulent flow," Chem. Eng. Commun. 158, 201 (1997).

${ }^{37}$ J. Abrahamson, "Collision rates of small particles in a vigorously turbulent fluid," Chem. Eng. Sci. 30, 1371 (1975).

${ }^{38}$ G.-D. Jin, G.-W. He, L.-P. Wang, and J. Zhang, "Subgrid scale fluid velocity timescales seen by inertial particles in large-eddy simulation of particle-laden turbulence," Int. J. Multiphase Flow 36, 432 (2010).

${ }^{39}$ Y. Zhou, A. S. Wexler, and L.-P. Wang, "On the collision rate of small particles in isotropic turbulence: II. Finite inertia case,” Phys. Fluids 10, 1206 (1998).

${ }^{40}$ Y. Zhou, A. S. Wexler, and L.-P. Wang, "Modelling turbulent collision of bidisperse inertial particles,” J. Fluid Mech. 433, 77 (2001). 